\title{
DEVELOPMENT OF VHF (240-270 MHZ) ANTENNAS FOR SOOP (SIGNAL QF OPPORTUNITY) RECEIVER FOR 6U CUBESAT PLATFORMS
}

\author{
A. T. Joseph ${ }^{1}$, M. Deshpande'2, P.E. O'Neill', and L. Miles ${ }^{3}$ \\ ${ }^{1}$ Hydrological Sciences Laboratory/617, Hydrospheric and Biospheric Sciences Directory, \\ NASA/Goddard Space Flight Center (GSFC), Greenbelt, MD 20771 USA, Tel: 301-614-5804, \\ Fax: 301 -614-5808, e-mail: Alicia.T.Joseph@nasa.gov, Peggy.E.ONeill@nasa.gov \\ ${ }^{2}$ Code 555, NASA/Goddard Space Flight Center (GSFC), Greenbelt, MD 20771 USA, e-mail: \\ Manohar.d.Deshpande@nasa.gov
}

${ }^{3}$ Code 564, NASA/Goddard Space Flight Center (GSFC), Greenbelt, MD 20771 USA, e-mail: Lynn.r.Miles@nasa.gov

\begin{abstract}
The main goal of this research is to design, fabricate, and test deployable VHF antennas for $6 \mathrm{U}$ Cubesat platforms to enable validation of root zone soil moisture (RZSM) estimation algorithms for signal of opportunity (SoOp) remote sensing over the $240-270 \mathrm{MHz}$ frequency band. The proposed work provides a strong foundation for establishing a technology development path for maturing a truly global direct surface soil moisture (SM) and RZSM measurement system (Figure 1) over a variety of land covers with limited density restrictions. In SoOp methodology, signals transmitted by already existing transmitters (known as transmitters of opportunity, in this case the Military Satellite Communication (MilSatCom) System's UHF Follow-On program) are utilized to measure properties of reflecting targets by recording reflected signals using a simple passive microwave receiver.
\end{abstract}

\section{Objectives}

(1) To develop and test a deployable VHF (240-270 MHz) antenna technology for SoOp receivers for $6 \mathrm{U}$ Cubesat platforms; (2) to perform measurement of SM and RZSM using the proposed antennas deployed on a ground-based SMAP simulator boom truck; and finally (3) to develop and validate the RZSM and SM estimation algorithms from the measured data (where RZSM is defined as the 
volumetric soil moisture contained in the top $1 \mathrm{~m}$ of the soil column). The proposed technology will help the maturation of a mission concept of a constellation of spaceborne Cubesats in Low Earth Orbit (LEO) (shown in Figure 1) for remote sensing of SM and RZSM using the MilSatCom System in Geosynchronous Earth Orbit (GEO) as transmitters of opportunity.

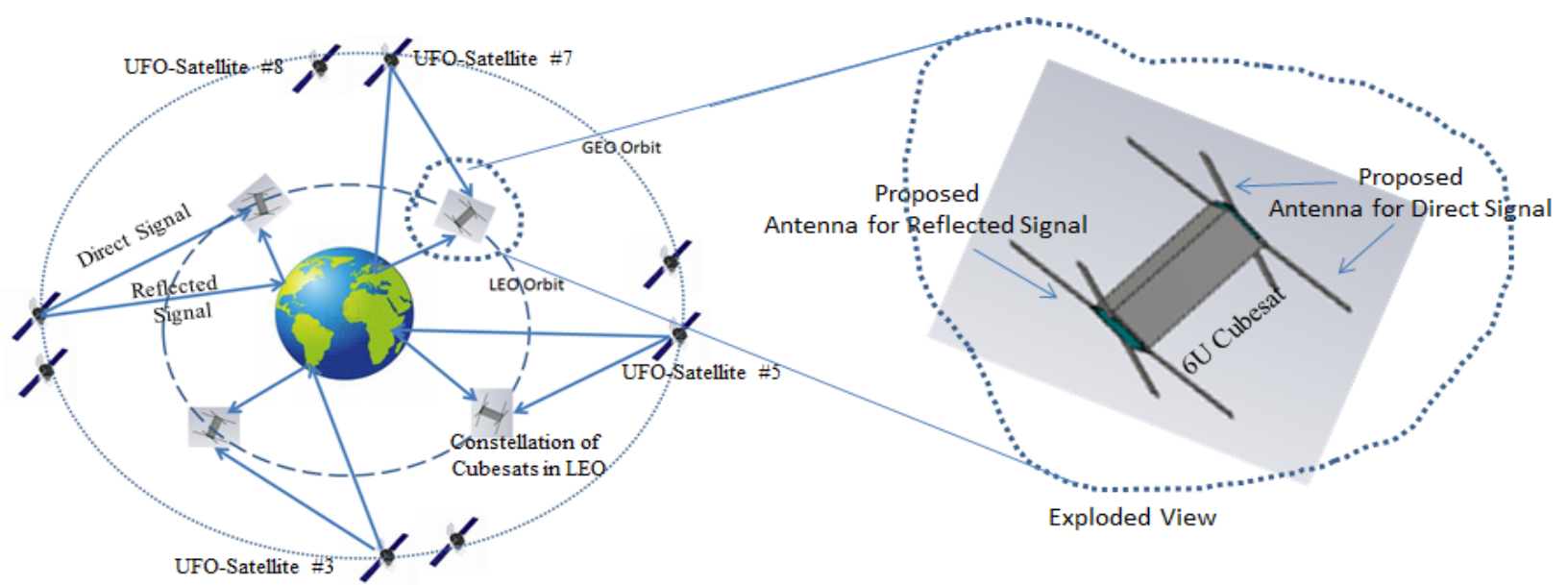

Figure 1: Artist's view of a constellation of spaceborne Cubesats in LEO for SM and RZSM measurements using UFO (Ultra High Frequency Follow-On)-Satellites in GEO as SoOp transmitters. The Ultra High Frequency Follow-On program is a constellation of eight communications satellites that provides tactical narrowband UHF satellite communications to DOD and other government agencies.

The proposed technology has the potential for forming the basis of an Earth Science mission using a constellation of spaceborne Cubesats for SM and RZSM measurements. The matured spaceborne SoOp VHF receiver technology can then be used to develop high-return Earth science missions for global measurement of SM and RZSM utilizing NASA's existing Earth Science flight opportunities (such as EVI, EVM).

GSFC is developing a 6U Cubesat platform technology (called "Dellingr") to provide data bus and communication links for GSFC science instruments, and which is planned to serve as a next generation platform for certain types of LEO science missions. Utilization of the 6U Cubesat platform context for the work proposed here will directly enable the eventual integration on future Cubesats of the antenna technology developed under this proposal without the need for additional redesign work. 
Knowledge of RZSM up to a depth of 1 meter and surface SM up to a depth of 0.05 meter on a global scale, at a spatial resolution of 1-10 km through moderate-to-heavy vegetation, is critical to understanding global water resources and the vertical moisture gradient in the Earth's surface layer which controls moisture interactions between the soil, vegetation, and atmosphere. Current observations of surface SM from space by L-band radiometers $(1.4 \mathrm{GHz})$ and radars $(1.26 \mathrm{GHz})$ are limited to measurements of surface SM up to a depth of $\sim 0.05$ meter through moderate amounts of vegetation. This limitation is mainly due to the inability of L-band signals to penetrate through dense vegetation and deep into the soil column. Satellite observations of the surface moisture conditions are coupled to sophisticated models which extrapolate the surface SM into the root zone, thus providing an indirect estimate rather than a direct measurement of RZSM. To overcome this limitation, low-frequency airborne radars operating at $435 \mathrm{MHz}$ and $118 \mathrm{MHz}$ have been investigated, since these lower frequencies should penetrate denser vegetation and respond to conditions deeper in the soil. However, these airborne observations are handicapped because of difficulties in getting permission from the FCC (Federal Communication Commission) and the NTIA (National Telecommunications and Information Administration) to radiate because of valid concern of radio frequency interference to existing users of these frequencies. Furthermore, because of their large weight, volume, and hardware and software complexity, extension of such systems to spaceborne missions would be prohibitively massive and expensive.

Developing bi-static reflectometry using VHF (250 MHz) geostationary satellite signals of opportunity creates the potential of directly observing SM and RZSM on a truly global basis from a constellation of small satellite-based receivers in low earth orbit. The technique provides the longwavelength (1.2 meter, 5 times larger than existing instruments) needed to remotely sense deeper into the root zone. It also overcomes spectrum management restrictions because the instrument approach is receive-only.

Preliminary link budget calculations at $250 \mathrm{MHz}$ with antenna gain of $2 \mathrm{~dB}$ and EIRP (Effective Isotropic Radiated Power) due to MilSatCom Follow-on transmitters equal to $29 \mathrm{dBw}$ show that the SNR (Signal to Noise Ratio) of the reflected signal is around $39 \mathrm{~dB}$, which is well above minimum detectable SNR (18 dB). This high SNR shows that it is feasible to detect reflected signals at LEO distances. Preliminary calculations show that using 8 Cubesats orbiting at a $500 \mathrm{~km}$ altitude will cover the latitude range of interest with a revisit time of 1 day. With a temporal resolution of 1 
day and a spatial resolution of $1.5 \mathrm{~km}$, the soil moisture information available from the Cubesat constellation would be a valuable data set for many hydrology applications at local to global scales.

\section{References}

J. Gunther, T. Moon, C. Swenson, C. Fish, Reliable space-to-earth communication as a secondary service in the $460-470 \mathrm{MHz}$ band. Int. J. Satell. Commun. Netw. (2014, in press)

N.C. Maynard, Electric field measurements in moderate to high density space plasmas with passive double probes, in Measurement Techniques in Space Plasmas Fields, ed. by R.F. Pfaff, J.E. Borovsky, D.T. Young (American Geophysical Union, Washington, 1998). doi:10.1002/9781118664391.ch2 